\title{
Tecnura
}

http://revistas.udistrital.edu.co/ojs/index.php/Tecnura/issue/view/640

DOI: http://dx.doi.org/10.14483/udistrital.jour.tecnura.2014.SE1.a08

INVESTIGACIÓN

\section{Metodología de evaluación de usabilidad de interfaces humano-máquina}

\section{Usability evaluation methodology of human-machine interfaces}

\author{
Luini Leonardo Hurtado Cortés*, Jhon Alejandro Forero Casallas**
}

Citation / Para citar este artículo: Hurtado Cortés, L. L., \& Forero Casallas, J. A. (2014). Metodología de evaluación de usabilidad de interfaces humano-máquina. Revista Tecnura, Edición especial, 103-113.

Fecha de recepción: 29 de noviembre de 2013 / Fecha de aceptación: 11 de julio de 2014

\section{RESUMEN}

Este artículo presenta la aplicación y validación de una metodología para la evaluación de la usabilidad de interfaces humano-máquina (IHM) en sistemas de supervisión industrial. Inicialmente, se presentan los aspectos fundamentales de evaluación de usabilidad de sistemas basados en software. Luego, se describe la metodología, que utiliza un sistema de adquisición de datos para supervisión y control de procesos (SCADA), un sistema de registro de eventos (EVENT LOGGER), un programa de clasificación de eventos en criterios de usabilidad, y un sistema adaptativo de inferencia neuro-difusa (ANFIS). La metodología fue aplicada a varias interfaces de operador para la monitorización de una planta. Finalmente, para comprobar la validez de la metodología se realizó una prueba de hipótesis frente a otras dos metodologías tradicionales: inspección por el experto e indagación a los usuarios. Se determinó que la metodología propuesta tiene equivalencia con las otras dos, pero tiene la ventaja de ser completamente automatizada. Los resultados permitirán ser retomados para su aplicación generalizada en el diseño de interfaces humano-máquina en cualquier sistema tecnológico para mejorar las prestaciones de la evaluación.

Palabras clave: Interfaz humano-máquina, sistemas de supervisión, sistemas neuro-difusos, usabilidad.

\section{ABSTRACT}

This paper presents the implementation and validation of an Usability Evaluation methodology of Human-Machine Interfaces (HMI) in industrial supervision systems. Initially, the basic aspects of Usability Evaluation of software-based systems are presented. Then, the methodology; which uses a Supervisory Control and Data Acquisition system (SCADA), an event log system (EVENT LOGGER), a classification program of events in usability criteria, and an Adaptive Neuro-Fuzzy Inference System (ANFIS) is described. The methodology was applied to various operator interfaces for plant monitoring. Finally, to check the validity of the methodology, a hypothesis test was performed against two other traditional methods: expert inspection and user inquiry. It was determined that the proposed methodology is equivalent to the other two, but its advantage is being fully automated. The results will be considered

\footnotetext{
* Ingeniero mecánico, especialista en automática e informática industrial, magíster en ingeniería-automatización industrial; doctor en ingeniería-automática. Docente, Universidad Distrital Francisco José de Caldas. Bogotá, Colombia. Contacto: Ilhurtadoc@udistrital.edu.co

** Ingeniero mecánico, especialista en automatización industrial, magíster en ingeniería-automatización industrial. Docente, Universidad Distrital Francisco José de Caldas. Bogotá, Colombia. Contacto: jaforeroca@udistrital.edu.co
} 
for widespread application in the design of HumanMachine Interfaces in any technological system to improve the performance of the evaluation.

Keywords: Human-Machine Interface, Neuro-fuzzy Systems, Supervision Systems, Usability.

\section{INTRODUCCIÓN}

Existen diversas perspectivas teóricas y empíricas que encierra el término usabilidad para sistemas interactivos. Para ello, en primer lugar se referencia desde lo expuesto por el estándar ISO 9241 de 1998, que describe los requisitos ergonómicos para trabajo de oficina con terminales de despliegue visual y explica algunos de los principios básicos subyacentes (ISO 9421, 1998). El estándar define la usabilidad como "la extensión para la que un producto puede ser usado por usuarios específicos, para lograr metas específicas con efectividad, eficacia y satisfacción en un contexto de uso específico"; además plantea aquellos factores que tienen un efecto en la usabilidad de productos software.

Según el estándar de Requisitos y Evaluación de Calidad de Productos de Software o SQuaRE (por sus siglas en inglés), la usabilidad es analizada en términos de su comprensibilidad, aprendizaje, operabilidad, atractividad y complacencia. En este estándar la usabilidad es un atributo de la calidad del software, siendo la calidad del software definida por el estándar como "un conjunto de atributos que se manifiestan por el esfuerzo necesario para el uso y por la valoración individual de tal uso por un conjunto de usuarios involucrados" (ISO 25000, 2005).

Para el caso de productos de software, se plantean teorías que centran los modelos en personas (Norman, 1986)(Wickens, 2012)(Anderson, 2012) y teorías de evaluación de usabilidad de las interfaces (Rodeiro, 2001)(Lorés, 2001)(Dix, 2004).

Por otra parte, se analiza la evaluación, la cual consiste en probar algo, tanto para saber si funciona correctamente como si no, si cumple con las expectativas o no, o simplemente para conocer cómo funciona una determinada herramienta o utensilio; la evaluación de la usabilidad es una actividad que comprende un conjunto de metodologías y técnicas que analizan la usabilidad de un sistema en diferentes etapas del ciclo de vida del software (Granollers, Lóres, \& Cañas, 2005). De la misma forma, se debe comprender que es necesario complementar la evaluación desde la perspectiva del usuario utilizando métodos de indagación y desde la perspectiva del experto, utilizando métodos de inspección. Un excelente trabajo de revisión sobre varios aportes al concepto de usabilidad y su evaluación se recoge en (Ankita \& Sanjay, 2012), allí, en una de las referencias se plantean diez factores de usabilidad y veintiséis criterios de evaluación(Seffah, Donayaee, Kline, \& Padda, 2006), que posteriormente son agrupados y plasmados en una taxonomía (Rios, Vásquez, Mosqueira, \& Moret, 2010).

Recientemente se ha hecho evidente la preocupación por la evaluación de la usabilidad y calidad del software de uso industrial. Se han propuesto enfoques mediante el uso de redes neuronales artificiales, que agilizan la evaluación en contextos particulares (Da Ponte \& Da Silveira, 2008), dado que existen dificultades a la hora de recoger los datos necesarios para analizar el comportamiento de los usuarios frente a las interfaces. Otros enfoques han sido el desarrollo de taxonomías para facilitar la identificación y recogida de las variables asociadas a los datos de interacción de los usuarios con el software, tales como rutas de navegación y agrupación de caracteres, y determinar así el comportamiento de los usuarios durante la interacción con la interfaz (Hanna, Richards, \& Jacobson, 2012). Igualmente, la utilización de la teoría inventiva para la resolución de problemas (TRIZ), que combina reglas del diseño de la interacción, métodos múltiples de evaluación de la usabilidad, recolectores de tendencias de la evolución de la interacción (Filippi \& Barattin, 2013), modelos formales de tareas basados en TaMoGo$\log$ (Rukh, 2012), la teoría de la actividad (Cao, Lijue, \& Shijian, 2013), y otros relacionados con la combinación de teoría del diseño y los sistemas 
de información en la interacción humano-computador (Spagnoletti \& Tarantino, 2013).

Sin embargo, desde el punto de vista de interfaces para monitorización y supervisión de plantas de procesos, aún son escasos los aportes sobre metodologías de evaluación de usabilidad, debido a la separación existente entre las operaciones automatizadas de los procesos y su interpretación a través de la interfaz para la toma de decisiones por parte del operador. Una aproximación para tal integración es la metodología de modos de marchas y paros o guía GEMMA (Ponsa, Vilanova, \& Díaz, 2007) que combina sistemas de eventos discretos DES y actividades humanas; así el operador humano puede cumplir un rol dentro de las acciones de los dispositivos automáticos sobre el proceso. La misma guía se aplica posteriormente sobre un simulador para el entrenamiento de operadores (Ponsa, Amante, \& Díaz, Evaluación de la usabilidad para la tarea de supervisión humana en sala de control industrial, 2009). Otra propuesta metodológica involucra nuevas tendencias en supervisión de procesos como aportes preliminares para la construcción de sistemas de supervisión holónicos (ente autónomo y cooperativo) que relacionan aspectos formales (modelos) con aspectos tecnológicos (interfaces de operador) (Zapata, Cardillo, \& Chacón, 2011), pero no plantea la manera de evaluar la usabilidad de tal conjunción.

Los enfoques anteriores están orientados a permitir al equipo de desarrollo de software recopilar y analizar automáticamente los usuarios y sus actividades y el comportamiento del sistema para el reconocimiento de problemas de usabilidad y problemas de funcionalidad de interfaces en forma eficiente y eficaz. Como consecuencia, se espera que las interfaces se ajusten mejor a la dinámica de los sistemas socio-técnicos en diferentes niveles (es decir, individual, grupal, organizacional, institucional, etc.), esto no es posible sin la aplicación de métodos que permitan soportar la evaluación. Los métodos de evaluación pueden clasificarse de varias maneras (Granollers, MPlu+a. Una metodología que integra la ingeniería del software, la interacción persona ordenador y la accesibilidad en el contexto de equipos de desarrollo multidisciplinares, 2004), como se resume en la tabla 1.

En cuanto al lugar de realización, se distinguen dos categorías generales:

- Los que se realizan en el laboratorio. El objetivo del evaluador, a veces, es comprobar aspectos sin que precise ayuda de los usuarios. Este tipo de comprobaciones las realiza, normalmente, en su entorno de trabajo, o sea, en el laboratorio, en donde puede además traer usuarios para un estudio particular.

- Los que se hacen en el entorno natural o habitual del escenario en donde se realizan las tareas por evaluar. Esta situación se produce cuando el evaluador hace su trabajo en el lugar natural en donde se realiza la escena, el lugar de interacción habitual del usuario.

En cuanto al tipo de técnica, se distinguen tres categorías:

- Métodos de inspección: su principal característica es que unos expertos examinan aspectos de la interfaz del sistema relacionados con la usabilidad y la accesibilidad que la misma ofrece a sus usuarios. Estos métodos tienen en cuenta las opiniones, juicios, informes de los inspectores sobre elementos específicos de la interfaz como factor fundamental de la evaluación de la usabilidad. Son populares en el ámbito de las empresas de producción de servicios software, pues permiten identificar, clasificar y contabilizar un gran número de errores potenciales de usabilidad a precio relativamente bajo.

- Métodos de indagación: la información acerca del agrado del usuario, quejas, necesidades e identificación de requisitos, son informaciones indispensables sobre todo en etapas tempranas del proceso de desarrollo para que proporcionen información relacionada con el uso y las posibilidades de acceso de un producto. Este tipo de métodos se realiza hablando con los usuarios, observándolos, usando 
el sistema en trabajo real, y obteniendo respuestas a preguntas verbalmente o por escrito.

- Métodos de prueba: En estos métodos, los usuarios representativos trabajan en tareas concretas utilizando el sistema (o el prototipo) y los evaluadores utilizan los resultados para ver cómo la interfaz de usuario da soporte a los usuarios con sus tareas.

En cuanto a la automatización, se pueden distinguir entre métodos:

- Automáticos: son aquellos métodos que disponen de mecanismos (hardware y/o software) que facilitan la comprobación de los aspectos por validar. Los métodos automáticos resultan altamente eficientes puesto que se realizan muy rápidamente y los resultados provienen de los propios parámetros, sin apreciaciones subjetivas. Una de las características que hacen más atractivos estos métodos es la posibilidad de realizar tests remotamente (Bartek \& Cheatham, 2012); (Dray \& Siegel, 2004) a través de internet. Su desventaja es que se usan en sistemas ya finalizados y no en etapas tempranas del ciclo de vida del desarrollo.

- Manuales: al contrario de los métodos anteriores, no disponen de mecanismos que automaticen su realización. Estos consumen más recursos: tiempo, evaluadores, etc., pero pueden evaluar aspectos que se "salen del patrón" y pueden realizarse en cualquier etapa del desarrollo y con cualquier clase de prototipo.

Según el tipo de participantes en la evaluación, se pueden distinguir los siguientes métodos:

- Con usuarios y/o implicados: Son los métodos en los que se evalúa el sistema con la intervención directa de usuarios representativos, pudiendo también participar en las sesiones personas que sin ser usuarios finales tienen la condición de implicados del sistema.

- Métodos sin usuarios y/o implicados: Estos métodos los aplican solo expertos evaluadores con ayuda de guiones, pautas o documentos que permiten hacer un seguimiento de los que han hecho los usuarios mientras utilizaban el sistema.

Tabla 1. Clasificación de los métodos de evaluación de la usabilidad.

\begin{tabular}{lc}
\hline & \multicolumn{1}{c}{$\begin{array}{c}\text { Clasificación de los métodos } \\
\text { de evaluación de usabilidad }\end{array}$} \\
\hline \multirow{2}{*}{ Lugar } & Laboratorio \\
\hline \multirow{2}{*}{ Técnica } & Entorno real \\
\cline { 2 - 2 } & Inspección \\
\hline Participantes & Indagación \\
\hline \multirow{2}{*}{ Automatización } & Con usuarios \\
\cline { 2 - 2 } & Sin usuarios \\
\hline
\end{tabular}

Fuente: Granollers, 2004.

De acuerdo con la anterior clasificación, en este trabajo la evaluación de la usabilidad de la interfaz de supervisión se realizó en un laboratorio, utilizando métodos de inspección por un experto e indagación a los usuarios y se convalidó con el método automático propuesto como se explica a continuación.

\section{MATERIALES Y MÉTODOS}

A. Lugar de realización: La metodología se aplicó en dos lugares, una sala de automatización de procesos que funciona como un entorno natural de supervisión de procesos y una sala especialmente equipada para efectuar evaluaciones como un laboratorio de usabilidad.

B. Participantes: para la evaluación de laboratorio se utilizaron 12 estudiantes distribuidos en tres grupos y para la evaluación en la sala de automatización, se utilizaron 3 operadores de salas de control de proceso. Los estudiantes, de los programas de ingeniería entre $8^{\circ}$. y $10^{\circ}$. semestres tienen competencias en procesos industriales relacionados con fluidomecánica, termotransferencia, 
máquinas, modelamiento, control y simulación de procesos industriales y automatización. Los operadores, por su experiencia trabajaron individualmente. El período de experimentación fue una hora. Además del diseño de la metodología, los autores de este trabajo actuaron como facilitadores de la evaluación.

C. Escenarios: Para la realización de las pruebas se utilizó un proceso de dosificado de líquidos, en donde es posible generar situaciones de manera programada y controlada; las condiciones del proceso cambian gradualmente, a través de la interfaz de supervisión, el operador debe identificar la situación y sus consecuencias antes de tomar las acciones correctivas necesarias.
D. Descripción de la metodología: La metodología de evaluación de Usabilidad de Interfaces Humano-Máquina desarrollada consta de un sistema de adquisición de datos para supervisión y control de procesos para el diseño de las pantallas de interacción con el operador, un sistema de registro de eventos de interacción, un programa que clasifica los eventos en criterios de usabilidad y un sistema de inferencia neuro-difuso adaptativo (ver la figura 1).

El sistema de adquisición de datos para supervisión y control que se utilizó fue una versión de demostración del paquete InduSoftWebStudio (IWS), que permite construir con todas las funciones del

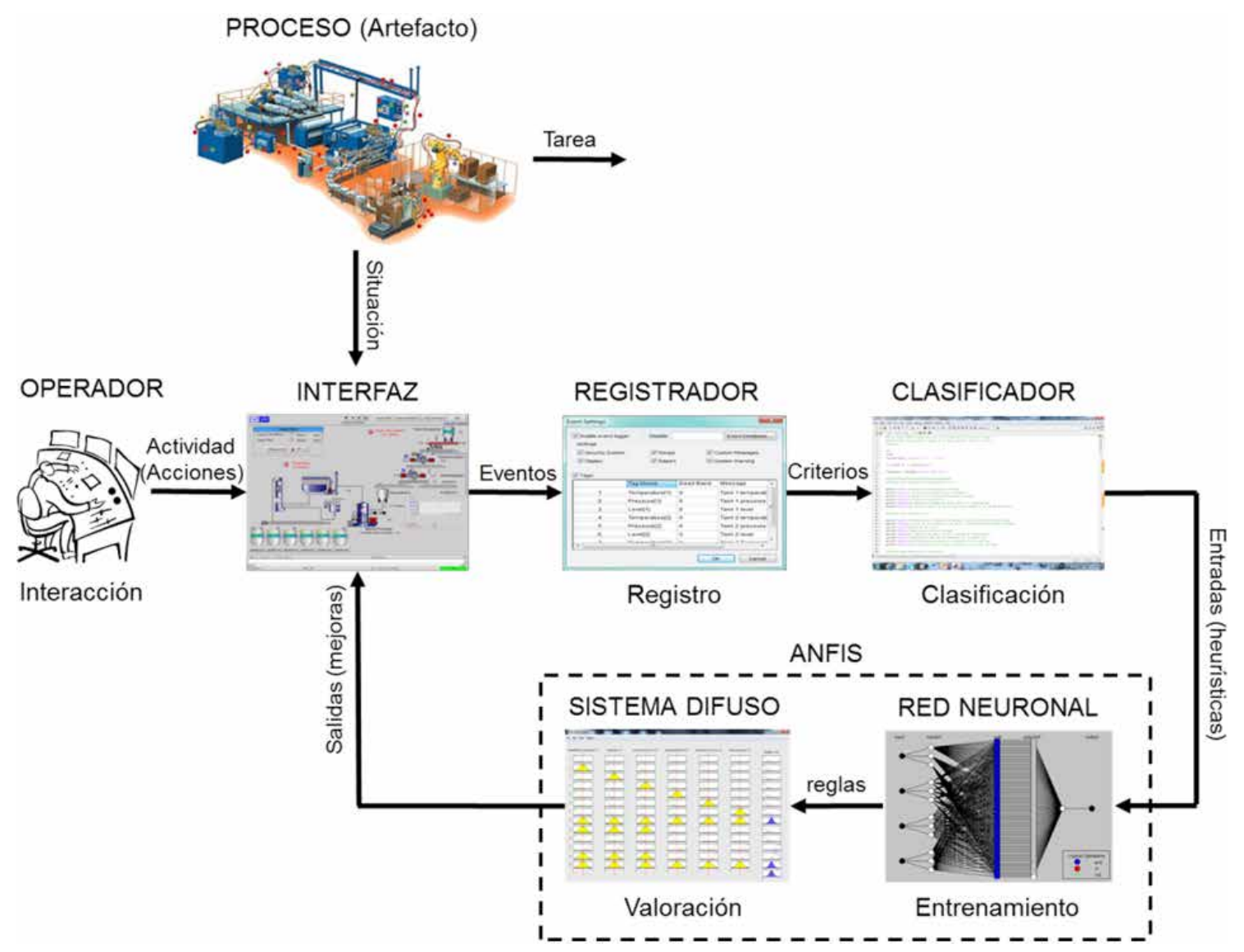

Figura 1. Esquema general del sistema de evaluación de usabilidad de Interfaces de Supervisión de Procesos.

Fuente: Elaboración propia. 
sistema de supervisión. Este paquete cuenta con una base de datos de etiquetas de proyecto para gestionar datos en tiempo de ejecución, incluyendo tanto variables internas como escaneado E/S para dispositivos sensores y accionamientos, controladores configurables para comunicarse en tiempo real con controladores lógicos programables, dispositivos E/S remotos y otros dispositivos de adquisición de datos, pantallas de interfaces animadas y cuadros de mando; y módulos opcionales, como registro de alarmas, registro de eventos, registro de tendencias, procedimientos, informes, lógica programable, programadores, un sistema de seguridad y una completa base de datos de interfaces. Con este paquete se desarrolló una interfaz de supervisión de un proceso de dosificado de líquidos, con varias pantallas como la que se muestra en la figura 2.

Por su parte, el registrador de eventos ofrece la trazabilidad de las acciones del operador durante la interacción con la interfaz o de la actividad interna de la aplicación, como cambios en la seguridad del sistema, operaciones de apertura/ cierre de pantallas, operaciones de ejecución de

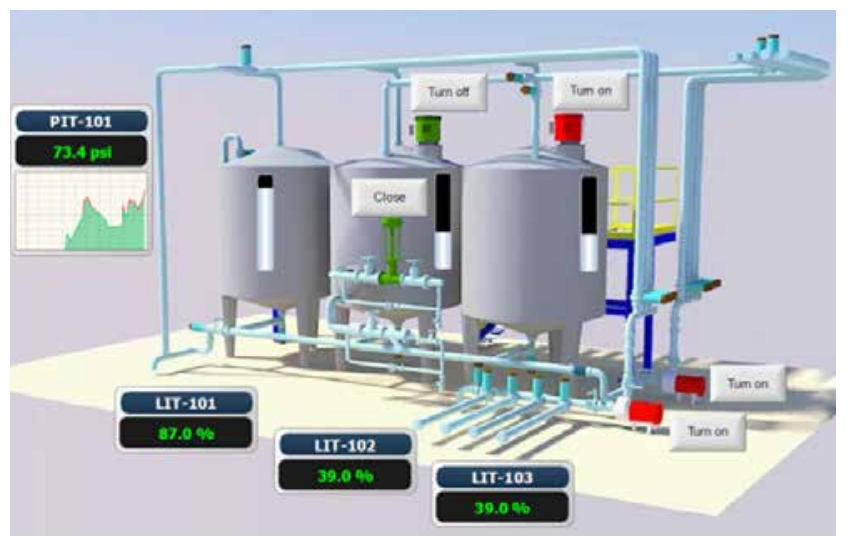

Figura 2. Pantalla de interfaz para la supervisión de procesos.

Fuente: (InduSoft, 2012). procedimientos o de informes, mensajes personalizados y alertas del sistema. Asimismo, cualquier cambio de valor de etiquetas, incluyendo mensajes personalizados (ver la figura 3).

Cada acción del operador tiene un código estandarizado como evento, el registro de los eventos es almacenado en archivos de la base de datos del IWS. Un programa explora los eventos correspondientes a las acciones del operador y los clasifica en diez criterios de usabilidad de interfaces definidos en(Seffah, Donayaee, Kline, \& Padda, 2006) (ver la tabla 2). Las acciones tomadas por el registrador de eventos constituyen una base de conocimiento. Los criterios son utilizados por el sistema adaptativo de inferencia neuro-difusa en forma de vectores. El sistema de inferencia utiliza la base de conocimiento heurístico de las acciones de los operadores de procesos para el entrenamiento de una red neuronal. El entrenamiento genera un sistema de inferencia difuso, en donde cada regla se establece de acuerdo con la distribución normal del número de acciones del operador durante la interacción con el proceso.

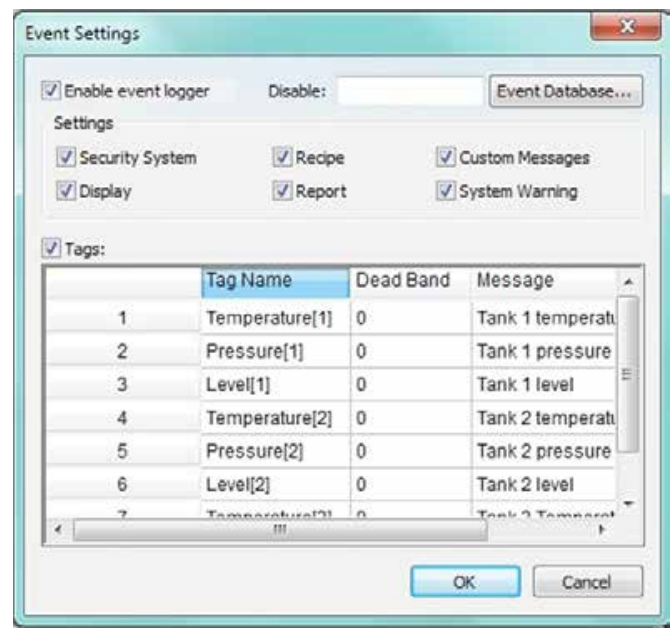

Figura 3. Módulo de registro de acciones del operador o de actividad interna de la aplicación.

Fuente: Elaboración propia. 
Tabla 2. Relación de las acciones del operador y los criterios de usabilidad.

\begin{tabular}{lll}
\hline \multicolumn{1}{c}{ Acciones del operador } & \multicolumn{1}{c}{ Eventos del sistema } & Criterios de usabilidad \\
\hline Reinicio del sistema & Account Logon y Logon/Logoff & Recuperación de errores \\
\hline $\begin{array}{l}\text { Seguimiento de una secuencia para el cumpli- } \\
\text { miento de una tarea }\end{array}$ & $\begin{array}{l}\text { Account Management/User account mana- } \\
\text { gement }\end{array}$ & Eficacia \\
\hline $\begin{array}{l}\text { Seguimiento de varias secuencias para el cum- } \\
\text { plimiento de varias tareas }\end{array}$ & Detailed Tracking & Productividad o multitarea \\
\hline $\begin{array}{l}\text { Verificación de la comunicación y actualiza- } \\
\text { ción de datos del proceso }\end{array}$ & DS Access & $\begin{array}{l}\text { Eficiencia o velocidad de } \\
\text { respuesta }\end{array}$ \\
\hline Activación de íconos dentro de las pantallas & Object Access & Accesibilidad o interactividad \\
\hline Tiempo transcurrido sin realizar acciones & Policy Change & Confianza \\
\hline $\begin{array}{l}\text { Activación de pantallas después de la aparición } \\
\text { de una señal de alarma }\end{array}$ & $\begin{array}{l}\text { Account Management/security group mana- } \\
\text { gement }\end{array}$ & Seguridad o fiabilidad \\
\hline Modificación de textos y color de pantallas & Privilege Use & Satisfacción o flexibilidad \\
\hline Modificación de objetos de pantallas & System & Coherencia \\
\hline
\end{tabular}

Fuente: Elaboración propia.

Para cada criterio de usabilidad se han definido tres funciones de pertenencia, baja, media y alta. En este caso se utilizó un sistema difuso que se basa en las reglas de Takagi-Sugeno, que usan una función lineal de las entradas al modelo como consecuente de las reglas. Tal estructura está en capacidad de representar una clase general de sistemas estáticos o dinámicos no lineales. La red neuronal se entrena con diez capas, cada capa con el número de neuronas correspondientes al número de entradas. La red neuronal utiliza el principio de retropropagación, en donde se ajusta el valor de los pesos en función del error generado. Esta técnica permite tener un método de optimización que se encuentra al definir el gradiente del error y minimizarlo con respecto a los parámetros de la red neuronal. El sistema completo es utilizado para determinar si la interfaz posee una alta, media o baja usabilidad.

E. Procedimiento: el sistema completo se ejecuta como una aplicación complementaria a la Interfaz humano-máquina durante la interacción con los operadores por un período de una hora. El sistema almacena los resultados de la evaluación que luego son comparados con los resultados de la evaluación por los métodos de inspección e indagación.

\section{RESULTADOS}

Para el entrenamiento de la red neuronal se utilizaron 100 datos, una capa oculta de 10 neuronas y el algoritmo de retro-propagación de Levenberg-Marquardt. Para comprobar la validez de la metodología automatizada (A), se efectuó una prueba de hipótesis frente a otras dos metodologías tradicionales: inspección por un experto (E) e indagación a los usuarios $(U)$. Se tabularon los datos de la experimentación de interacción entre los operadores y las interfaces IHM de supervisión de un proceso de control de flujo, nivel, temperatura y presión de líquidos. Las valoraciones arrojadas por el sistema desarrollado sirvieron para clasificar las interfaces como de baja, media y alta usabilidad.

Con los datos obtenidos se desea suponer que por las valoraciones dadas, los métodos son aproximados. Entonces la hipótesis nula es que en promedio las valoraciones son iguales o estadísticamente existen diferencias significativas que permitan afirmar que el promedio de los tres métodos no es el mismo. Para probar esta hipótesis se elaboraron tablas de Anova.

En la tabla de Anova la fuente de variación tiene dos componentes, el factor y el error. Esta 
fuente de variación se descompone en una suma de cuadrados, la suma de cuadrados del factor, expresada como una diferencia de cuadrados y la suma de cuadrados del error, otra diferencia de cuadrados. Los grados de libertad del factor es $\mathrm{C}-$ 1 y los grados de libertad del error es $n-c$, donde $c$ es el número de columnas (3) y $n$ es el numero de datos (30). Igualmente, se calculó el cuadrado medio como la suma de cuadrados entre el grado de libertad, tanto para el factor como para el error. En la última columna se calcula la estadística de prueba $f$, como el cuadrado medio del factor entre el cuadrado medio del error (ver la tabla 3 ).

Haciendo las operaciones y tomando de una tabla de valores críticos de distribución $f$ para $5 \%$ de área de rechazo, se ubica la relación entre 3 grados de libertad para el factor y 27 grados de libertad para el error; así se obtiene un valor crítico de 2,95. La estadística de prueba para la usabilidad baja es 0,09 , para media es 2,01 y para alta es 0,38 . Esto indica que la hipótesis no se descarta, es decir, en promedio las valoraciones son las mismas. Con

Tabla 3. Operaciones de Anova.

\begin{tabular}{lcccc}
\hline Fuente & Suma de cuadrados & Grados de libertad & Cuadrado medio & $\mathbf{f}$ \\
\hline Factor & SCfactor $=\sum \frac{C_{i}^{2}}{k_{i}}-\frac{\left(\sum x\right)^{2}}{n}$ & $c-1$ & CMfactor $=\frac{\text { SCfactor }}{c-1}$ & CMfactor \\
Error & SCerror $=\sum x^{2}-\sum \frac{C_{i}^{2}}{k_{i}}$ & $n-c$ & CMerror $=\frac{\text { SCerror }}{n-c}$ & CMerror
\end{tabular}

Fuente: Elaboración propia.

Tabla 4. Anova para usabilidad baja.

\begin{tabular}{lcccc}
\hline Fuente & Suma de cuadrados & Grados de libertad & Cuadrado medio & f \\
\hline Factor & 0,40 & 3 & 0,13 & \multirow{2}{*}{0,09} \\
\hline Error & 38,14 & 27 & 1,41 & \\
\hline
\end{tabular}

Fuente: Elaboración propia.

Tabla 5. Anova para usabilidad media.

\begin{tabular}{lcccc}
\hline Fuente & Suma de cuadrados & Grados de libertad & Cuadrado medio & f \\
\hline Factor & 6,69 & 3 & 2,23 & 2,01 \\
\hline Error & 29,95 & 27 & 1,11 & 2 \\
\hline
\end{tabular}

Fuente: Elaboración propia.

Tabla 6. Anova para usabilidad alta.

\begin{tabular}{lcccc}
\hline Fuente & Suma de cuadrados & Grados de libertad & Cuadrado medio & f \\
\hline Factor & 0,77 & 3 & 0,26 & \multirow{2}{*}{0,38} \\
\hline Error & 18,32 & 27 & 0,68 & \\
\hline
\end{tabular}

Fuente: Elaboración propia. 
ello, se deduce que la metodología propuesta tiene equivalencia con dos metodologías tradicionales, la inspección de un experto y la indagación a los usuarios, pero tiene la ventaja de que es completamente automatizada. Las tablas de Anova 4, 5 y 6 muestran los cálculos y la estadística de prueba obtenidos para los datos de la evaluación de usabilidad baja, media y alta de las interfaces.

Es importante aclarar que esta metodología está en función de la interacción con el operador, pero el comportamiento del operador puede obedecer a otras variables no tenidas en cuenta en la metodología. Por ejemplo, los operadores pueden manifestar diversos comportamientos o realizar varias actividades que no están ligadas directamente con el proceso, incluso la actividad de interacción con las pantallas de una interfaz puede generar aburrimiento entre los operadores. Estos elementos, aunque importantes, no se consideran dentro de esta metodología.

El desempeño del operador durante la interacción a través de la interfaz tiene una relación directa con el conocimiento que tenga del proceso, haciéndolo más eficaz o menos productivo a la hora de efectuar una tarea y/o tomar decisiones. Varias de las tareas asignadas a los operadores se rigen bajo ciertos patrones de secuencias a seguir, luego depende del operador y su habilidad para memorizar y realizar $n$ veces la misma acción, la cantidad de veces que se siga la misma secuencia en determinado tiempo. Los trabajos futuros están orientados a utilizar la metodología aquí presentada en varias aplicaciones, incluso de otros campos diferentes a la supervisión de procesos industriales, dado que actualmente existe un mercado diverso de productos con un alto grado de interacción con el usuario. Igualmente, para el proceso de evaluación de usabilidad de interfaces y/o productos interactivos es importante la incorporación de buenas aplicaciones informáticas para la captura y análisis de registro de datos; por ello, este también es un campo de exploración.

\section{CONCLUSIONES}

La usabilidad se reconoce como un factor de calidad importante para sistemas interactivos incluidas las interfaces de usuario de una gran variedad de dispositivos. El diseño de aplicaciones, de modo que logren efectivamente sus propósitos en términos de facilidad de uso, no es una tarea fácil, ante la existencia de varios métodos y técnicas para la evaluación de la usabilidad que no ha sido posible integrarlas en un sencillo marco conceptual que facilite su uso por los desarrolladores. La metodología desarrollada hace parte de las técnicas automatizadas de acuerdo con la clasificación presentada en este artículo. Sin embargo, incorpora a los usuarios (operadores) para extraer información durante la interacción en tiempo de ejecución en actividades de supervisión de procesos, lo cual constituye una innovación frente a técnicas tradicionales del mismo tipo.

La literatura tiende a definir la usabilidad en términos excesivamente breves y ambiguos y describe su aplicación en términos informales. Esta es una de las razones principales por las cuales predominan los diferentes enfoques y orientaciones en el estudio de la usabilidad. Aunque no existe una concepción universal sobre criterios reales de evaluación de la usabilidad de sistemas interactivos, con la aproximación presentada por varios autores se logró establecer una relación entre las acciones del operador sobre la interfaz y los eventos de interacción establecidos en el software de supervisión de procesos.

Con la estadística de prueba se determinó que la metodología es eficiente para actividades de interacción con interfaces de usuario, porque utiliza elementos que se encuentran incorporados y probados en el software de supervisión y se complementan con una técnica de inteligencia artificial que igualmente ha mostrado sus bondades en otras aplicaciones.

El objeto de la evaluación de usabilidad de interfaces es utilizar los resultados para realizar una 
retroalimentación, con el fin de mejorar los diseños y, por ende, la interactividad con el usuario. La metodología desarrollada le permite a un inexperto en usabilidad agilizar la evaluación para proponer las mejoras que den lugar a la optimización de la interacción con los procesos.

\section{AGRADECIMIENTOS}

Los resultados hacen parte del proyecto "Desarrollo de una metodología de evaluación de usabilidad de Interfaces Humano-Máquina (IHM) para la mejora del proceso de toma de decisiones en tareas de supervisión industrial", financiado por la Universidad Nacional de Colombia, Sede Manizales.

\section{FINANCIAMIENTO:}

\author{
Universidad Nacional de Colombia, Sede \\ Manizales.
}

\section{REFERENCIAS}

Anderson, J. R. (2012). Cognitive Psychology and its Implications. New York, USA: Worth Publishers.

Ankita, M., \& Sanjay, K. (2012). Usability Evaluation Methods: A Literature Review. International Journal of Engineering Science and Technology, 4(2), (2012)., 590-599.

Bartek, V., \& Cheatham, D. (1 de septiembre de 2012). IBM, DeveloperWorks. Obtenido de: Experience remote usability testing, part 1: Examine study results on the benefits and downside of remote usability testing: http://www.ibm.com/developerworks/ library/wa-rmusts1

Cao, X.; Lijue, W., \& Shijian, L. (2013). Research on Contextual Design in Human-Computer Interaction Under the Framework of Activity Theory. Proceedings of the 2012 International Conference on Information Technology and Software Engineering (pp. 853-868). Beijing: Springer-Verlag.

Da Ponte, M., \& Da Silveira, A. (2008). A Methodology for Evaluation the Usability of Software for Industrial Automation Using Artificial Neural Networks:
Case Study-Eletrobrás. International Conference on Computational Intelligence for Modeling, Control and (pp. 430-435). Vienna: IEEE.

Dix, A. F. (2004). Human-Computer Interaction. Madrid, España: Pearson.

Dray, S., \& Siegel, D. (2004). Remote possibilities?: international usability testing at a distance. Interactions, 10-17.

Filippi, S., \& Barattin, D. (2013). Integrating Systematic Innovation, Interaction Design, Usability Evaluation and Trends of Evolution. En: A. Chakrabarti, Sustainable product development, CIRP Design 2012 (pp. 301-311). London: Springer-Verlag.

Granollers, T. (2004). MPlu+a. Una metodología que integra la ingeniería del software, la interacción persona ordenador y la accesibilidad en el contexto de equipos de desarrollo multidisciplinares. Lleida, España: Universidad de Lleida.

Granollers, T.; Lóres, V., \& Cañas, J. (2005). Diseño de sistemas interactivos centrados en el usuario. Barcelona, España: Editorial UOC, Colección Informática.

Hanna, N.; Richards, D., \& Jacobson, M. (2012). Automatic Acquisition of User Models of Interaction to Evaluate the Usability of Virtual Environments. Lectures Notes in Computer Science, 43-57.

InduSoft (1 de septiembre de 2012). InduSoft Web Studio. Obtenido de: InduSoft Web Studio, Product Features: http://www.indusoft.com/

ISO 25000 (2005). ISO 25000, Calidad del producto software. Navarra, España: International Organization of Standarizations.

ISO 9421 (1998). Requisitos ergonómicos para trabajos de oficina con pantallas de visualización de datos (PVD), Parte 11: Guía sobre usabilidad. Navarra, España: International Organization of Standarization.

Lorés, J. J. (2001). Introducción a la Interacción PersonaOrdenador. Lleida, España: Jesús Lorés.

Norman, D. (1986). Cognitive engineering. New Jersey, EUA: Norman \& Draper.

Ponsa, P.; Amante, B., \& Díaz, M. (2009). Evaluación de la usabilidad para la tarea de supervisión humana en sala de control industrial. Revista RIAI, Revista 
Iberoamericana de Automática e Informática Industrial, 84-93.

Ponsa, P.; Vilanova, R., \& Díaz, M. (2007). Introducción del Operario Humano en el Ciclo de Automatización de Procesos Mediante la Guía GEMMA. Información Tecnológica, 21-30.

Ríos, D.; Vásquez, A.; Mosqueira, E., \& Moret, V. (2010). Usability: A Critical Analysis and a Taxonomy. Ríos, D., Vásquez, A., Mosqueira, E. y Moret, V. International Journal of Human Computer-Interaction, 53-74.

Rodeiro, J. (2001). Representación y Análisis de la componente visual de la interfaz de usuario. Tesis doctoral. Vigo, España: Universidad de Vigo.

Rukh, H. (2012). Incorporating Usability Evaluation in Software Development Environments. KI-künstliche Intellinez, 197-200.
Seffah, A.; Donayaee, M.; Kline, R., \& Padda, H. (2006). Usability measurement and metrics: A consolidated model. Software Quality Control, 159-178.

Spagnoletti, P., \& Tarantino, L. (2013). User Centered Systems Design: The Bridging Role of Justificatory Knowledge. En: R. Baskerville, M. De Marco, \& P. Spagnoletti, Designing Organizational Systems. Lectures Notes in Information Systems and Organization (pp. 105-121). Berlin: Springer-Verlag.

Wickens, C. (2012). Engineering Psychology and Human-Computer Performance. New Jersey, EUA: Pearson.

Zapata, G.; Cardillo, J., \& Chacón, E. (2011). Aportes Metodológicos para el Diseño de Sistemas de Supervisión de Procesos Continuos. Información Tecnológica, 97-114.

\section{(c) $(1) \Theta \Theta$}


